\title{
Application of an optimization-based curtailment service provider in real-time simulation
}

\author{
Omid Abrishambaf, Pedro Faria and Zita Vale*
}

\author{
* Correspondence: zav@isep.ipp.pt \\ GECAD - Research Group on \\ Intelligent Engineering and \\ Computing for Advanced \\ Innovation and Development, \\ Institute of Engineering - \\ Polytechnic of Porto (ISEP/IPP), Rua \\ Dr. António Bernardino de Almeida, \\ 431, 4200-072 Porto, Portugal
}

\begin{abstract}
The use of demand response programs and distributed renewable energy resources are intensively discussed. These concepts play a key role in the distribution network, especially smart grids and microgrids. Nowadays, most of the implemented demand response programs are considered for large-scale resources, which make small and medium resources unable to participate in electricity market negotiations. In order to overcome this barrier, a third-party entity, namely an aggregator, can be considered as an intermediate player between the demand side and grid side. For this purpose, curtailment service provider is considered as an aggregator, which aggregates small and medium-scale resources, who do not have adequate capacity of reduction or generation and allow them to participate in wholesale electricity markets as a unique resource. However, before massive implementation of business models, the performance of the curtailment service provider should be adequately surveyed and validated in order to prevent future problems. This paper proposes a real-time simulation model of a curtailment service provider, which employs several real and laboratory hardware equipment considered as hardware-in-the-loop in the real-time simulator. Furthermore, an optimization problem is developed for a curtailment service provider in order to optimally schedule the available resources including several demand response programs and distributed renewable resources, aiming at minimizing its operation costs. The implemented case study considers a distribution network with 20 consumers and prosumers, and 26 renewable-based producers including wind and photovoltaic generation, where the developed model is performed in real-time for $12 \mathrm{~min}$ and behaviors of small and medium prosumers and producers is surveyed.
\end{abstract}

Keywords: Demand response, Curtailment service provider, Real-time simulation, Hardware-in-the-loop, Optimization

\section{Background}

The daily increment of electrical energy usage impels the network operator to provide efficient solutions regarding energy resources. Demand Response (DR) programs and Distributed Generation (DG) are two major concepts, which play a key role in this context (Aghaei and Alizadeh 2013). DR programs can be defined as the modification of the consumption in demand side, according to the price variations or financial incentive paid by DR managing entity to the consumers (Hurtado et al. 2018). In fact, by promoting DR programs, all the players can benefit. The demand side will be

(c) The Author(s). 2018 Open Access This article is distributed under the terms of the Creative Commons Attribution 4.0 International License (http://creativecommons.org/licenses/by/4.0/), which permits unrestricted use, distribution, and reproduction in any medium provided you give appropriate credit to the original author(s) and the source, provide a link to the Creative Commons license, and indicate if changes were made. 
encouraged to utilize DR programs for reducing their electricity bills, and the grid side will benefit from that by reducing congestion of the grid and lowering the consumption in the peak periods (Falvo et al. 2014). DR programs are categorized into two main groups (Mortaji et al. 2017; Shariatzadeh et al. 2015):

- Price-based: in which the end-users modify their consumption based on the electricity price variations. Real-Time Pricing (RTP) is an example of this group;

- Incentive-based: in which the grid operator pays a remuneration to the end-users in exchange for modifying their consumption pattern. Direct Load Control (DLC) is an example of this group.

If the use of DR programs is merged with Distributed Renewable Energy Resources (DRERs), the grid operator would be able to fully benefit from these concepts and participate in market negotiations (Wang et al. 2015). However, both DR and DRERs should have enough capacity of reduction and generation in order to participate in the market negotiations. Based on several surveyed references (Bakr and Cranefield 2015; Khezeli et al. 2017; Paterakis et al. 2017), the minimum reduction capacity of customers for participating in DR programs in several electricity markets is $100 \mathrm{~kW}$. This means that in such markets it is not possible for small and medium consumers, such as typical residential customers, to contribute to market negotiation individually (Gkatzikis et al. 2013). In order to overcome this barrier, a third party entity can be considered as a solution in order to aggregate small and medium scale resources and represent them as a unique resource in the energy market negotiations with adequate capacity (Siano 2017; Reddy 2016). Curtailment Service Provider (CSP) is a concept that can be considered as a third party in the electrical network operation (Hillman 2011; Faria and Vale 2013). However, before the implementation of business models, it is required to test and validate the model concepts in reliable and physical simulation platforms, which are capable to provide actual measurement and control in order to identify future problems (Mao et al. 2018). For this purpose, the use of fully computational resources to simulate an electrical distribution network can be very difficult and unaffordable, and perhaps the produced results will be far from the reality (Olivares et al. 2014). Therefore, a real-time simulation strategy would be a satisfying solution for integrating both reality and simulation results (Alvarez-Gonzalez et al. 2017).

There are few research works concerning the real-time simulation of a CSP model; this was a motivation for the present work. This paper provides a real-time simulation model for a CSP by using several real and laboratory hardware equipment considered as Hardware-In-the-Loop (HIL). The CSP aggregated resources include consumers, producers, and prosumers who do not have adequate capacity of generation and DR reduction in order to participate in the wholesale market negotiation, therefore, they establish a contract with CSP in order to be aggregated and managed by this third party. Moreover, an optimization problem is developed in this paper in order to be used by CSP for optimal resources scheduling, which aims at minimizing its operation costs. The HIL equipment employed for CSP resources contain two small and medium scale laboratory loads, laboratory wind turbine and Photovoltaic (PV) emulators, and a real PV producer, which are controlled and managed by a real-time simulation machine (OP5600) through MATLAB/Simulink environment. In this way, the main purpose of 
the paper is to demonstrate that the hybrid simulation platform is also capable to implement optimal scheduling results and DR programs.

The rest of paper is organized as follows: Section 2 presents the related works and clarifies the main contribution of this paper. Section 3 explains the presented CSP model including all DR programs and mathematical formulation regarding the optimization problem. Section 4 presents the real-time simulation model considered for the CSP, where all HIL infrastructures and MATLAB/Simulink models implemented in the OP5600 are denoted. Section 5 discusses a case study implemented using the presented model in order to test and validate the system capabilities, and its results are presented in Section 6. Finally, the main conclusions of the work are presented in section 7 .

\section{Related works}

There are several research works related to this topic. In (Bottaccioli et al. 2017a) the authors presented a hybrid simulation platform, including a PV simulator and a real-time network simulation model. The PV simulator models the real PV systems and calculates the energy generation based on the input data such as the solar radiation. For the network model, it has been used the OP5600 real-time simulator, in order to verify the PV model in the real conditions, such as real electricity network configurations. It has been presented a case study for testing the platform on the actual electricity network conditions in Turin, Italy, and the results demonstrated that the current configuration of the network cannot handle the integration of a significant number of PV installation, therefore, an improvement is necessary for preventing future problems. Although the authors in (Bottaccioli et al. 2017a) used OP5600 for a hybrid platform, they did not focus on the aggregation level and optimal resource scheduling, and also, there was not real or laboratory consumer loads in order to emulate the consumption profile in real-time.

In (Bottaccioli et al. 2017b), a novel and flexible platform has been developed in real-time simulator OP5600. In this work, the authors integrated load simulation and physical devices by employing an Internet of Things (IoT) adaptor. The proposed simulation platform contains PV and storage integration; it can be used for testing and validating the smart grids concepts. They also presented a case study that utilizes the developed platform in the real conditions of the network in northern Italy, and they confirmed the performance of the system. However, in this work, the authors did not include any optimization algorithm integrated with OP5600 regarding the aggregator concepts.

Reference (Marulanda et al. 2014) utilized a realistic methodology by considering CSP as a load aggregator in order to evaluate the impact of DR programs in the day-ahead Colombian electricity market. They considered that CSP can bid in the wholesale market by using the demand-bidding program. They also presented a tool for the market operator in order to quantify the impact of DR programs on the system. Realistic values from Colombian market have been used in order to perform numerical tests. The numerical results shown that the penetration of demand-bidding program changes the dispatch for different generation units. Although they utilized real input data for the model, the final results are totally numerical without any experimental test and validation. 
Reference (Rotger-Griful et al. 2016) introduced a co-simulation platform, called virtual integration laboratory (VirGIL) with HIL devices in order to evaluate DR programs in a residential building located in Denmark. This platform is able to control the ventilation system of the building, as well as to integrate power system simulation, communication, and control. In the case study, the authors surveyed the impacts of a DR program defined by the CSP on the controlling of the ventilation system in the building using VirGIL. The results demonstrated the capabilities of the developed model, in which the ventilation systems can track the changes with 1-min time interval in order to perform the decisions at the certain time. However, they focused on a particular consumer on the CSP network, and they do not describe how the CSP perform the optimization and the day-ahead scheduling of the resources in order to define DR programs.

In (Li et al. 2017), the authors presented a real-time operation scheme for thermostatically controlled loads aggregation in electricity markets, which is considered as biding resource contributing in the day-ahead market negotiations. Moreover, they utilized an optimization model in order to maximize the profit of the aggregator in the regulation market. They performed several experimental tests with the proposed load aggregation simulator as a load shedding service provider, which validated the system. Although, they provided only the numerical results, and they did not use any real-time simulator or any HIL equipment in order to validate their system with actual measurements and control for gaining realistic results.

The main focus of the present paper is to implement a real-time simulation platform for an optimization-based CSP model considering several real and laboratory HIL infrastructures, which are controlled based on the optimal resource scheduling of a CSP and DR programs implementation. The HIL equipment enables the system to validate the developed methodology by using real data and enables actual measurements and control of hardware devices that are outside the simulation environment. The scientific contribution of this paper is to address an optimization based CSP model to the small and medium scale consumers and producers by employing a hybrid simulation platform, including real systems, laboratory emulators, and mathematical models.

\section{CSP model}

This section concerns the presented CSP model applying DR programs to its consumers, and the optimization formulations utilized by the CSP in order to optimally schedule the consumption and generation resources.

\section{Model description}

As it was mentioned before, in order to implement a DR program in a network, there is a minimum capacity for load reduction, which should be reached by the customers in order to be able to participate in the DR event. In this context, if a consumer has enough capacity of reduction, He can directly establish a contract with the system operator or DR managing entity. However, for small and medium consumers, who do not have adequate capacity of reduction, a third party entity (CSP in this paper) should aggregate these consumers, and allow them to participate in DR event as one. This means that the small and medium consumers can establish a DR contract with a CSP 
in order to be able to utilize DR programs, and participate in the wholesale market negotiations. The overall concept of the proposed CSP model is illustrated in Fig. 1.

As it can be seen in Fig. 1, the CSP is an intermediate entity between the demand side and grid side. In the demand side there are several small and medium scale consumers, producers, and prosumers (a consumer who can also produce); the CSP will be responsible for aggregation, scheduling and remuneration of DR events. In the grid side, the CSP will be in charge of market negotiations, energy trades, and bids with grid players, such as market or system operator. Also, the CSP should be able to accommodate the uncertainty that is related to the actual consumption and generation and to the actual response of consumers to DR events. In some cases, the CSP can make Direct Load Control (DLC) in the consumers loads but in other cases, without control hardware, the forecasting tools are very relevant in order to take full potential of the DR event. Such aspects should be considered in the scheduling and remuneration phases of the method since it doesn't imply with the HIL simulation model, only with the parameters of each simulation.

The network considered for the CSP model (right side of Fig. 1) is an internal low voltage distribution network of a university campus in Porto, Portugal (Silva et al. 2015). The CSP grid includes 21 bus with underground cables, where a MV/LV transformer in BUS \#21 connects the CSP grid to the main network. This CSP network is considered as a part of the main network containing 220 consumers, and 68 DG units (left side of Fig. 1), which has been developed by the authors in the scope of previous works (Abrishambaf et al. 2017).

The CSP is able to perform the resources scheduling considering external suppliers, DG units (especially renewable producers and surplus of prosumer generation), and also DR programs. For this purpose, the customers that intend to participate in DR events can establish a contract with the CSP in three different programs: Direct Load Control (DLC), Load Reduction (Red.), and Real-Time Pricing (RTP). The characteristics of these DR programs are shown in Table 1. The load shifting program is an

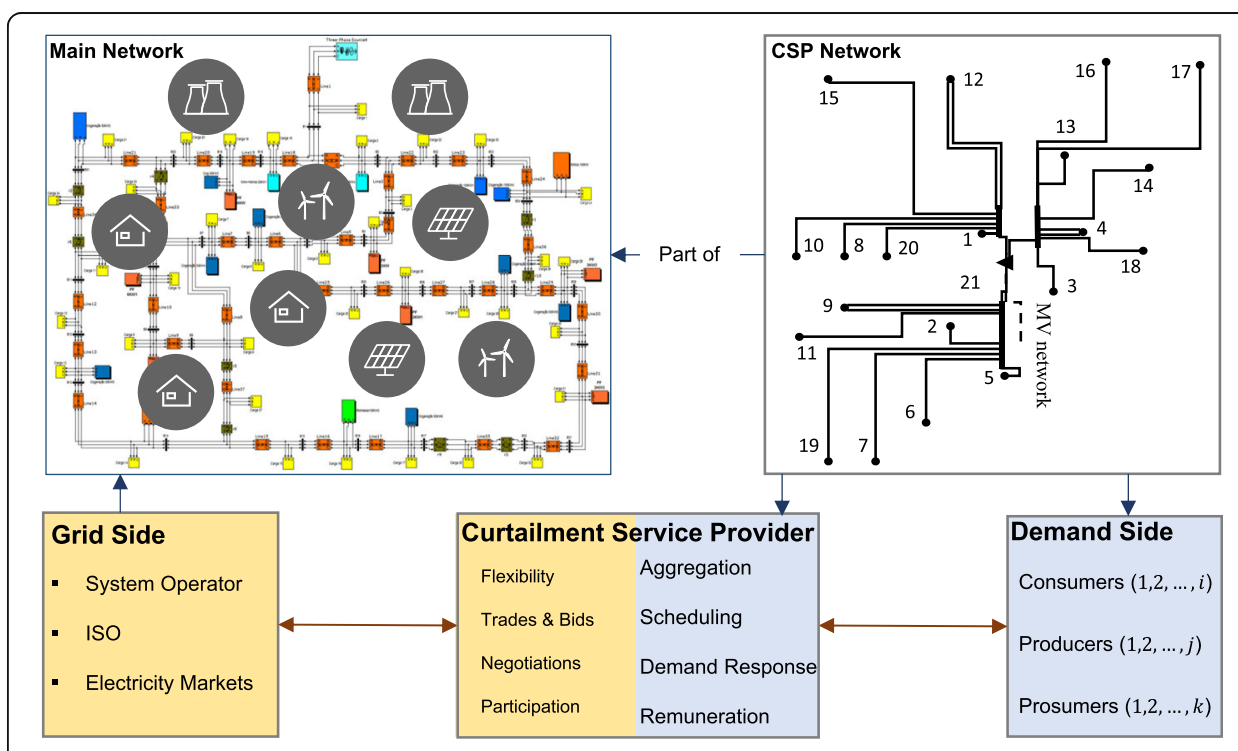

Fig. 1 The overall architecture of CSP model 
Table 1 DR Programs proposed by the CSP to customers

\begin{tabular}{lllll}
\hline DR Type & Mandatory/Noluntary & Remuneration & Activation/Signal & Measure/Contract \\
\hline DLC & Mandatory & Cost/kWh reduced & DLC per equipment & Actual kWh reduction \\
Red. & Voluntary & Cost/kWh reduced & Reduction notification & Actual kWh reduction \\
RTP & Voluntary & N/A & Electricity price notification & N/A \\
\hline
\end{tabular}

important tool for the CSP since it can manage the consumption and shift it based on the available rate of generation. A load shifting model has been developed by the authors in the scope of their previous work (Faria et al. 2015), and since the purpose is to show that the developed model can also implement DR programs, three different programs have been presented in this paper and load shifting is not considered.

If a customer establishes a contract with the CSP for DLC, He will give permission to the CSP to directly control the devices whenever it witnesses with critical periods, for instance, while He faces a technical or economic reason. For this purpose, the customer will be notified about the event, and receive remuneration based on the actual kWh reduction. If a customer makes a contract with CSP for Red. program, while the CSP decides to apply DR event, the customer will be notified for consumption reduction, and if it is accepted, he receives remuneration based on kWh reduction. Finally, if a customer has a RTP contract, it specifies a value of electricity price somehow that if the electricity price raises and is greater than that specific value, He will decrease the consumption as much as it is specified in the contract. This means that the customer will be notified about the real-time electricity price, and if $\mathrm{He}$ agrees to participate, $\mathrm{He}$ will reduce the consumption. In the RTP program, the customer will not earn remuneration for the consumption reduction, which means RTP program has no cost from CSP standpoint.

In total, the CSP can perform resource scheduling and aggregation processes considering DG units, external suppliers, and DR resources. In this context, the CSP should utilize an optimization problem in order to optimally manage the resources, which will be demonstrated in the next sub-section.

\section{Optimization problem}

The CSP always tends to supply the demand using the local resources. Renewable resources are the first ones that CSP utilizes for scheduling since these have a lower price from the CSP standpoint. After that, DR resources, especially RTP program, are the next options for CSP scheduling, and external suppliers would be the last choices for the CSP since it is considered as the most expensive resources from the CSP standpoint. Therefore, an optimization problem is required for the CSP in order to provide an optimal resource scheduling. However, as previously stated, the optimization problem is not a core contribution of the paper since the main focus of the paper relies on demonstrating that the hybrid simulation platform is also capable to implement optimal scheduling results and DR programs.

The objective function presented for CSP to minimize his Operation Cost (OC) is shown by Eq. (1). In this model, it is considered that technical verification of network is 
the responsibility of the network operator, and the CSP is not accountable for such matters.

\section{Minimize}

$$
\begin{aligned}
O C=\sum_{i=1}^{I}\left(\sum_{s=1}^{S}\left(P_{E x t(s, i)} \times C_{E x t(s, i)}\right)\right. & +\sum_{c=1}^{C s}\left[P_{D G(c, i)} \times C_{D G(c, i)}+P_{D L C(c, i)} \times C_{D L C(c, i)}\right. \\
& \left.\left.+P_{\operatorname{Red}(c, i)} \times C_{\operatorname{Red}(c, i)}+P_{R T P(c, i)} \times C_{R T P(c, i)}\right]\right)
\end{aligned}
$$

There are several constraints considered for this optimization problem. The first constraint stands for load balance, as shown in Eq. (2). The second constraint, in eq. (3), concerns DG units and prosumers, where indicates if the customer is a prosumer, its DG generation supplies the local demand first, and then, if there is any generation surplus, it will be injected to the CSP network.

$$
\begin{aligned}
& \sum_{s=1}^{S} P_{E x t(s, i)}+\sum_{c=1}^{C s}\left(P_{D G(c, i)}+P_{D L C(c, i)}+P_{\operatorname{Red}(c, i)}+P_{R T P(c, i)}\right) \\
& =P_{\operatorname{Load}(i)} \forall i \in\{1, \ldots, I\} \\
& P_{D G(c, i)}=\left\{\begin{array}{lr}
P_{D G(c, i)}-P_{\operatorname{Cons}(c, i)} & P_{D G(c, i)}>P_{\operatorname{Cons}(c, i)} \\
0 & P_{D G(c, i)} \leq P_{\operatorname{Cons}(c, i)}
\end{array} \quad \forall c \in\{1, \ldots, C s\}, \forall i \in\{1, \ldots, I\}\right.
\end{aligned}
$$

Additionally, the technical limitation of each resource should be considered in the optimization problem. For this purpose, eq. (4) and eq. (5) demonstrate the limitation regarding external suppliers and DG units, respectively.

$$
\begin{aligned}
& 0 \leq P_{E x t(s, i)} \leq P_{E x t(s, i)}^{\max } \quad \forall s \in\{1, \ldots, S\}, \forall i \in\{1, \ldots, I\} \\
& 0 \leq P_{D G(c, i)} \leq P_{D G(c, i)}^{\max } \quad \forall c \in\{1, \ldots, C s\}, \forall i \in\{1, \ldots, I\}
\end{aligned}
$$

The technical limitation regarding DR programs, including DLC, Red., and RTP programs, is shown by eq.(6-8), respectively.

$$
\begin{array}{ll}
0 \leq P_{D L C(c, i)} \leq P_{D L C(c, i)}^{\max } & \forall c \in\{1, \ldots, C s\}, \forall i \in\{1, \ldots, I\} \\
0 \leq P_{\operatorname{Red}(c, i)} \leq P_{\operatorname{Red}(c, i)}^{\max } & \forall c \in\{1, \ldots, C s\}, \forall i \in\{1, \ldots, I\} \\
0 \leq P_{R T P(c, i)} \leq P_{R T P(c, i)}^{\max } & \forall c \in\{1, \ldots, C s\}, \forall i \in\{1, \ldots, I\}
\end{array}
$$

In sum, the mathematical formulation of the CSP model for resource scheduling with the objective of minimizing the operation costs, are shown in this section. In the next section, this formulation will be implemented in the real-time simulation model considered for the CSP.

\section{Real-time simulation}

In this section, the real-time simulation model considered for the CSP is presented. Several real and laboratory hardware equipment is employed in order to simulate the model in real-time considering the HIL methodology.

The real-time simulation model implemented for the CSP is shown in Fig. 2. In this model, it is considered that there are 20 consumers and prosumers, and 26 producers. 


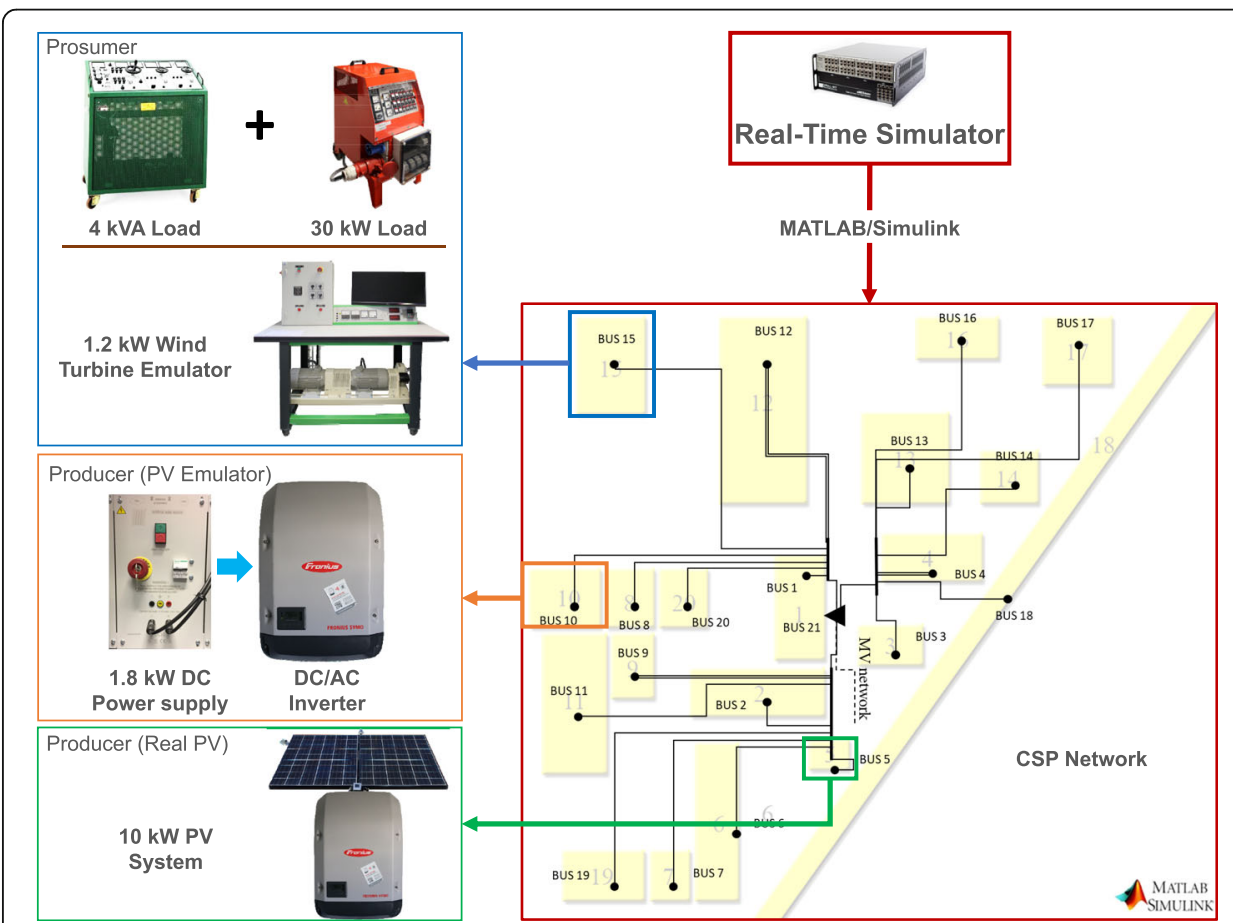

Fig. 2 Real and laboratory HIL equipment in MATLAB/Simulink CSP model

The consumers are 12 residential buildings, 6 commercial buildings, and 2 industrial units. Also, the producers are all renewable resources including $22 \mathrm{PV}$ systems and 4 wind generators. Moreover, external suppliers are considered.

As Fig. 2 shows, the CSP network has been modeled in MATLAB/Simulink in OP5600. In this network, several real and laboratory equipment has been employed in order to emulate the consumption and generation profiles of the CSP players via the HIL methodology. BUS \#15 is considered as a prosumer, BUS \#10 is dedicated to a $1.8 \mathrm{~kW}$ PV emulator, and BUS \#5 includes a real $10 \mathrm{~kW}$ PV producer.

For the prosumer in BUS \#15, a $4 \mathrm{kVA}$ and a $30 \mathrm{~kW}$ load emulate the consumption, and a $1.2 \mathrm{~kW}$ wind turbine emulator is responsible for wind generation. Moreover, for the two PV producers, in BUS \#10, a $1.8 \mathrm{~kW}$ DC power supply connected to a DC/AC inverter emulates a PV producer, and in BUS \#5, a real installation of PV system with a maximum capacity of $10 \mathrm{~kW}$ stands for another PV producer. All this equipment is controlled and monitored via OP5600 in MATLAB/Simulink.

Regarding the equipment connected to BUS \#15 (prosumer), in a $30 \mathrm{~kW}$ load, there are four relays that increase or decrease the rated consumption, and in a $4 \mathrm{kVA}$ load, there is an Arduino (www.arduino.cc), which manages the amount of consumption. The relays in $30 \mathrm{~kW}$ load are connected to Digital Output board of OP5600, and Arduino $^{\circ}$ has been connected to OP5600 via Ethernet interface, with MODBUS TCP/IP protocol. Also, in wind turbine emulator, there is an induction motor coupled with the generator, in which the motor emulates the wind turbine. The motor has a speed controller unit, which manages the speed of the wind, and therefore, the output power generation of the machine. The speed controller unit of this machine is connected to the Analog Output of OP5600. More information about these resources is available in 


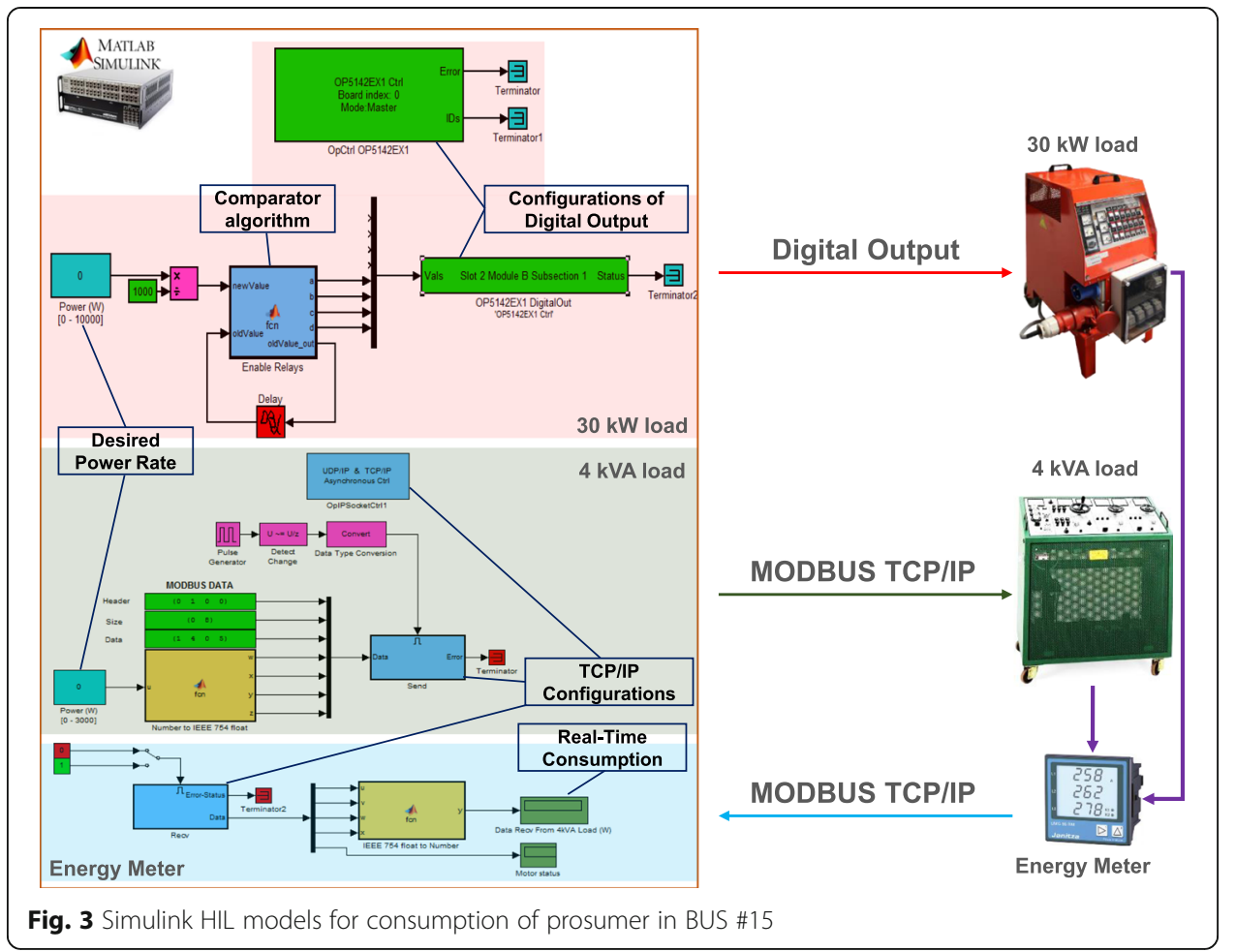

(Abrishambaf et al. 2015). Figures 3 and 4 present the Simulink models, implemented in OP5600 for controlling these resources via HIL.

As it can be seen in Fig. 3, two Constant blocks in Simulink indicate the desired consumption to be consumed by the $30 \mathrm{~kW}$ and the $4 \mathrm{kVA}$ loads. In the case of $30 \mathrm{~kW}$ load, the output of Constant block will be divided into the four binary outputs through a comparator algorithm in order to be provided to the Digital Output board. In the case of $4 \mathrm{kVA}$ load, the output of Constant block will be converted to MODBUS TCP/ IP format with IEEE 754 standard, which is four hexadecimal numbers. Furthermore, there is an energy meter for these two loads, which measures the consumed active power and transmit it to OP5600 in real-time via MODUS TCP/IP. By this way, OP5600 is able to transmit the favorable amount of power to the loads and simultaneously receives the real-time consumption of them.

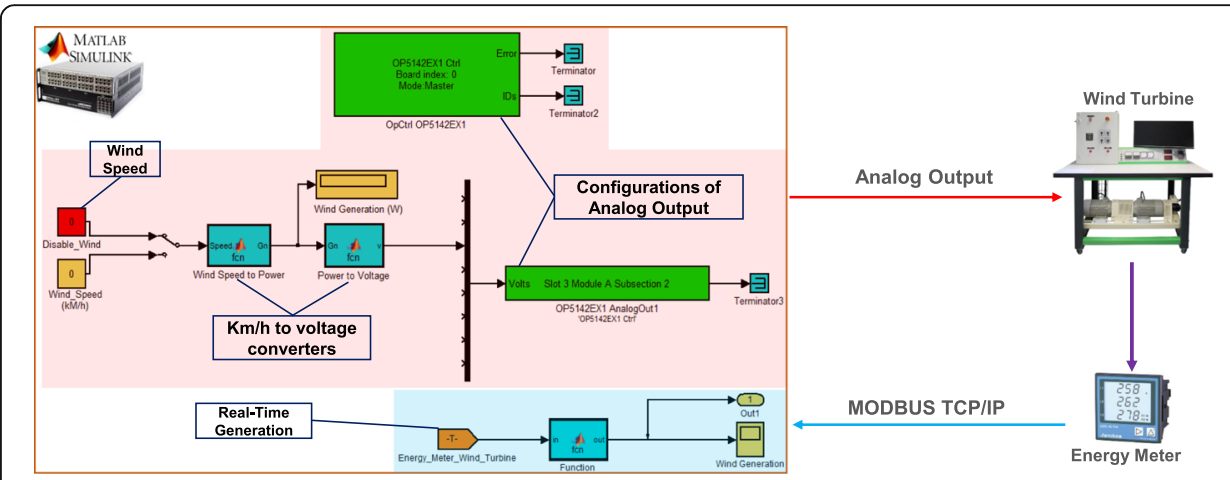

Fig. 4 Simulink HIL models for wind generation of prosumer in BUS \#15 
The Simulink model regarding the wind turbine emulator is shown in Fig. 4. In this model, since the wind turbine is controlled by Analog Output of OP5600, the wind speed data should be converted to a 0 to $+10 \mathrm{~V}$ signal. The output of a Constant block, which is favorable wind speed value, is converted to 0 to $+10 \mathrm{~V}$ voltage range through two developed algorithms implemented in two MATLAB Function blocks, and therefore, OP5600 controls the emulator based on the provided voltage. Simultaneously, an energy meter measures the generation of the emulator and transmit it to the OP5600 in real-time via MODBUS TCP/IP. The TCP/IP configurations blocks regarding the energy meter are not shown in Fig. 4 since these are similar to the ones in Fig. 3.

Regarding the $1.8 \mathrm{~kW}$ PV emulator located in BUS \#10, an Arduino ${ }^{\circ}$ has been utilized in the DC power supply in order to manage the output power between 0 and $100 \%$ of capacity. In fact, the DC power source simulates the PV arrays, which provides DC voltage, and the DC/AC inverter is a usual model that is utilized in real PV installations. The Simulink model for controlling and monitoring the equipment in BUS \#10 is shown in Fig. 5. In this model, two groups of TCP/IP blocks have been employed, one for the DC power source (Arduino ${ }^{\circ}$ ), and the other for the DC/AC inverter. By this way, the OP5600 transmits the desired value of PV generation to an Arduino via a MODBUS TCP/IP request and receives the real-time AC power generation from the $\mathrm{DC} / \mathrm{AC}$ inverter.

The Simulink model considered for real $10 \mathrm{~kW}$ PV producer in BUS \#5 is the same as the model presented in Fig. 5, however, the difference is that there is no DC power source, and therefore, OP5600 has no control over the PV generation and is only able to monitor the real-time $\mathrm{AC}$ power generation of the unit. This has been implemented via a group of TCP/IP blocks that request the real-time $\mathrm{AC}$ power generation from the $\mathrm{DC} / \mathrm{AC}$ inverter.

In sum, the Simulink models implemented in the real-time simulator (OP5600) have been demonstrated in this section. All Simulink models have been designed by relying on the HIL methodology in order to control real hardware resources and utilize real data in Simulink.

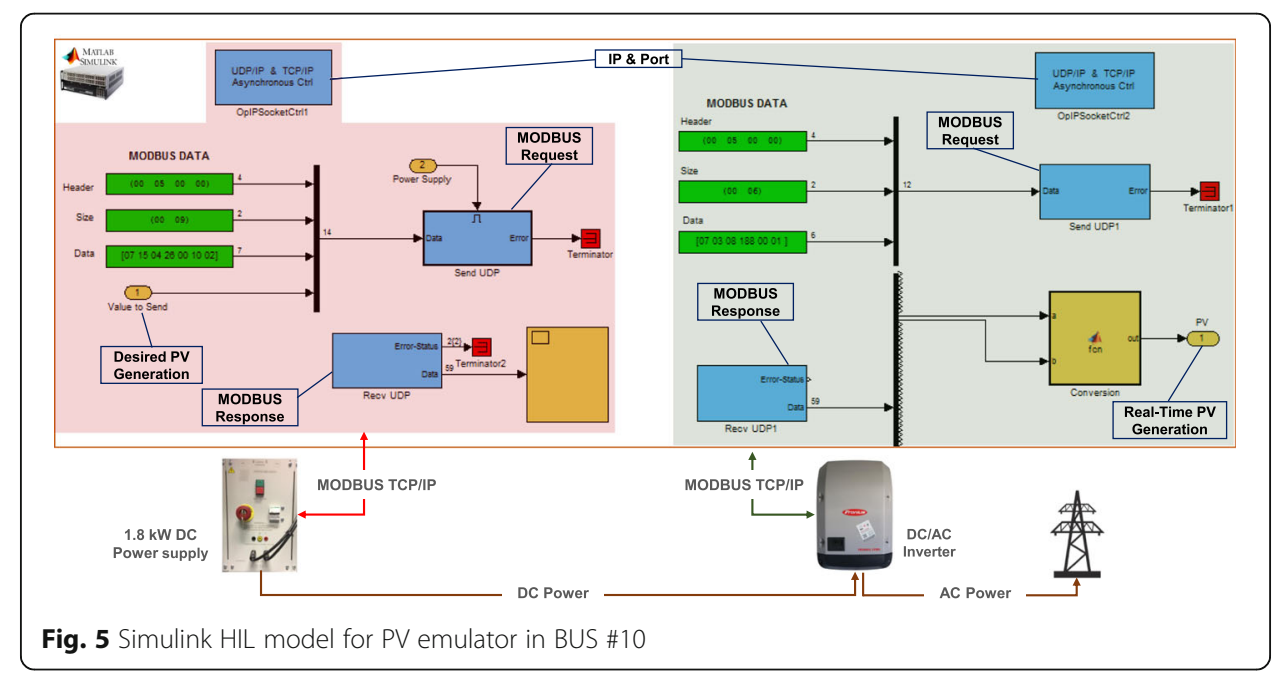




\section{Case study}

In this section, a case study tests and validates the developed Simulink models in OP5600. For this purpose, 24 periods of $30 \mathrm{~s}$ (12 min in total) are considered for running the model in real-time and obtain the results. The $12 \mathrm{~min}$ period, which is a rather short one, has been selected in order to provide deeper focus on the results analysis. Longer periods can be easily implemented using this hybrid platform. The consumption and generation profiles considered for the day-ahead scheduling of the CSP are shown in Fig. 6.

As Fig. 6a illustrates, in some periods the CSP is able to manage the consumption relying on local DG and DR resources, however, in other periods CSP is forced to purchase energy from external suppliers. The aggregated profiles shown in Fig. 6b, are related to the prosumer in BUS \#15, and to two PV producers in BUS \#10 and \#5, which are real data of GECAD research center in Porto, Portugal, adapted from GECAD database. The profiles shown in Fig. 6 are day-ahead profiles, which enable the CSP to manage and schedule the consumption and the generation of the network for the next day. By this way, the customers will be notified of the scheduled events for next day, and therefore, they have adequate time in order to negotiate and response their availability for the next day events.

In this case study, DG units are considered as the cheapest resource for CSP so these will be the first resource to be utilized by CSP to supply the demand. In the meantime, the price of buying energy from external suppliers is considered as the most expensive resource of the CSP. This means that, in high consumption periods, it is affordable for CSP to perform DR programs to reduce the consumption in order to avoid purchasing energy from the external suppliers. In this context, it is considered that the prosumer in BUS \#15 has established a DR contract with the CSP for $2.5 \mathrm{~kW}$ reduction between periods \#8 to \#19.

With this in mind, it is obvious that the CSP should perform the proposed optimization algorithm, in order to optimally schedule the resources for minimizing its operation costs. In this model, the real-time simulation starts from the OP5600 and Simulink model. Then, JAVA Application Programming Interface (API) is employed in order to transmit the Simulink data to the R Studio tools (www.rstudio.com), where the optimization algorithm has been implemented. Therefore, the optimization algorithm is performed, and afterward the optimized data including optimal resource scheduling results is transferred to the OP5600 and Simulink model using JAVA API. Full details regarding this process are available in (Abrishambaf et al. 2017), which has been developed by the authors in the scope of their previous works.

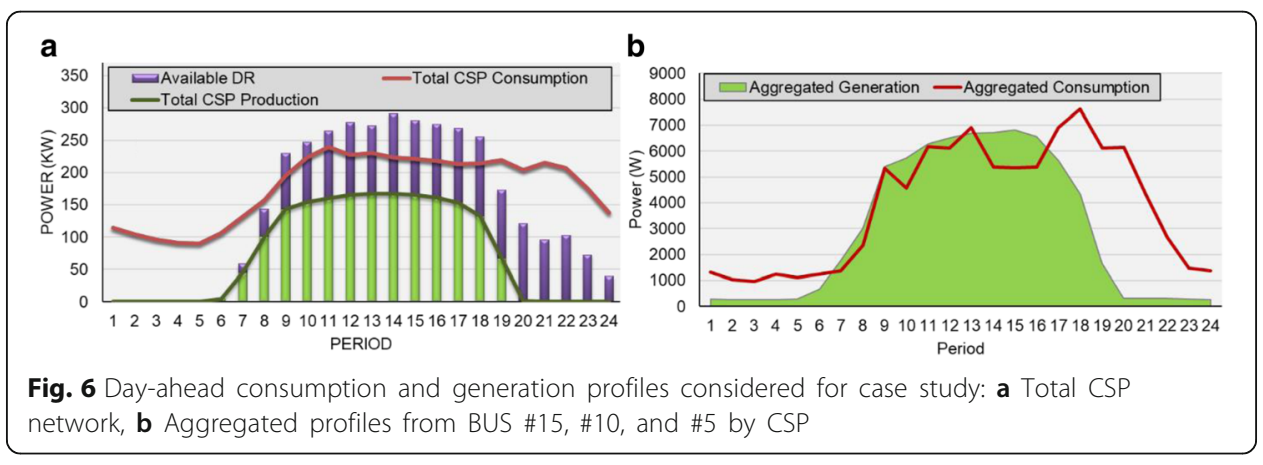


The optimization problem developed in this paper is a linear problem and is solved using R Studio. Since R studio tools play the role of mathematical problem solver and are separate from the real-time simulator, OP5600 is able to manage HIL devices according to the acquired results of any optimization model supported by $\mathrm{R}$ Studio, such as linear or nonlinear problems.

In other words, the outcomes of the optimization process, which is the economic resource scheduling, is the rate of power that has been requested from each consumer and generator in the CSP network. In order to implement these optimal scheduling results on the real resources, the conditions of the electrical network are important. The OP5600 real-time simulator used in this paper enables us to address this fact; we can validate the actual amount of reduction in the consumers and actual generation of energy resources and obtain the actual measurement results in order to be employed in the remuneration phase.

\section{Results}

In this section, at first, the results obtained from optimization problem are provided, and then, the real-time data acquired by OP5600 from Simulink is presented. The optimization results belong to all the CSP network, however, the main focus is given to the prosumer in BUS \#15, and to the two producers in BUS \#10 and \#5. Figure 7 illustrates the optimal resource scheduling results of the CSP for network players in BUS $\# 15$, \#10, and \#5.

As it can be seen in Fig. 7a, all DG units are responsible to provide the maximum generation to the CSP network since it is the cheapest resource of the grid. Additionally, Fig. 7b shows the load reduction in the scope of Red. DR program, which CSP utilized for prosumer in BUS \#15, for reducing the consumption in order to avoid purchasing energy from external suppliers.

When these optimization results are obtained, they are provided to the OP5600 real-time simulator as inputs. Consequently, the real-time simulator starts to control and manage the HIL equipment in order to implement the optimization results in real-time. The consumption profiles shown in Fig. 7b will be emulated by the $4 \mathrm{kVA}$ and the $30 \mathrm{~kW}$ loads; the wind production curve shown in Fig. 7a will be provided to the $1.2 \mathrm{~kW}$ wind turbine emulator; the PV profile in BUS \#10 will be modeled by the $1.8 \mathrm{~kW}$ PV emulator; and the PV curve in BUS \#5 will be the real profile of GECAD PV production. The output of the optimal energy resource scheduling model is a requested amount of power for each resource to manage its demand or generation in a

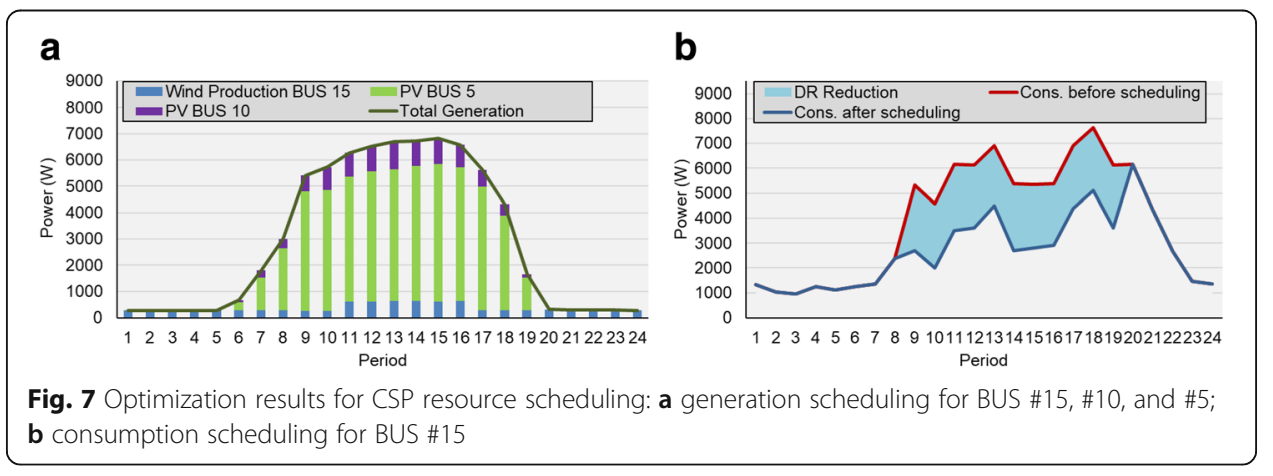




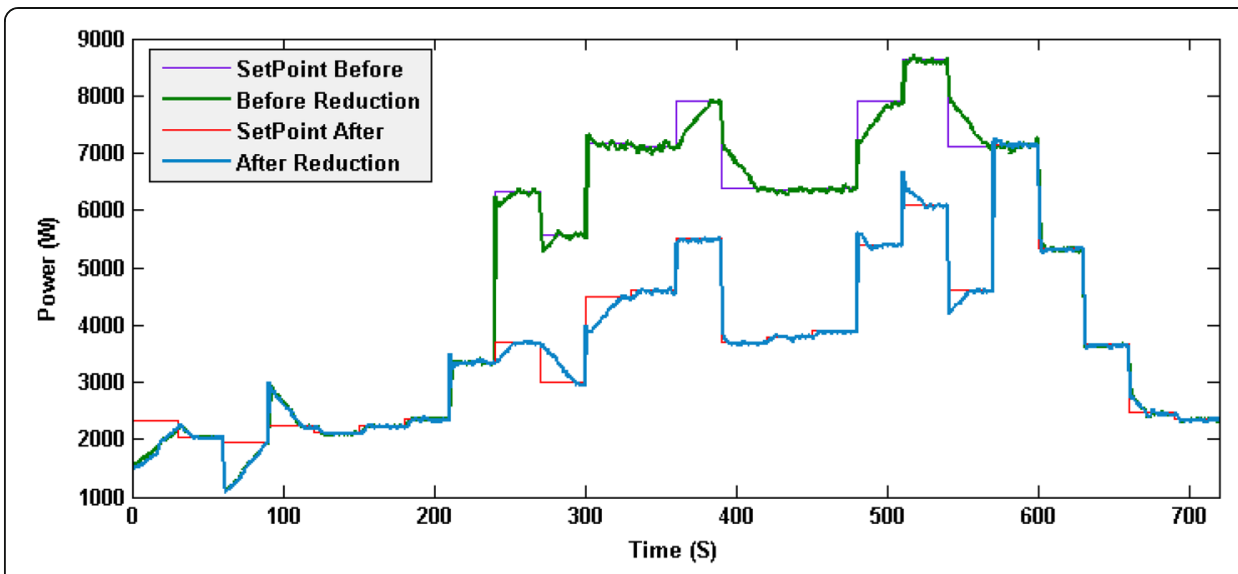

Fig. 8 Real-time simulation of consumption in BUS \#15 before and after DR reduction

certain period. However, the actual implementation of the demand reduction and the DG production requested for each real resource will depend on the electrical grid conditions. In fact, this is one of the advantages of using real-time simulation (OP5600) and laboratory equipment, as HIL, for modeling consumption and generation profiles. With this method, we validate the actual demand reduction, and the actual DG production in order to be used in the simulation results, namely for remuneration and aggregation goals.

Figures $8,9,10,11$ and 12 show the final results of the real-time simulation for $12 \mathrm{~min}$. All the results illustrated in these figures are adapted from OP5600 and MATLAB/Simulink. In this model, the time step for real-time simulation is configured as $0.5 \mathrm{~s}$. This means that OP5600 transmits the optimization results to HIL devices with $30 \mathrm{~s}$ time interval (one value for each period), and then, it acquires the real-time data with a $0.5 \mathrm{~s}$ time interval. The results shown in Figs. 8, 9, 10, 11 and 12 are the behaviors and reactions of customers during the scheduled events; they accepted their availability for these events in the day before.

In Fig. 8, the set point values are the ones that OP5600 transmitted to the $4 \mathrm{kVA}$ and to the $30 \mathrm{~kW}$ loads in order to simulate the consumption of the prosumer in BUS \#15. The green and blue lines are the responsibility of the loads in real-time. The DR event has been applied between the instant of $240 \mathrm{~s}$ (period \#8) to 570 (period \#19), which leads to reducing the consumption. As it can be seen in Fig. 8, whenever the rate of

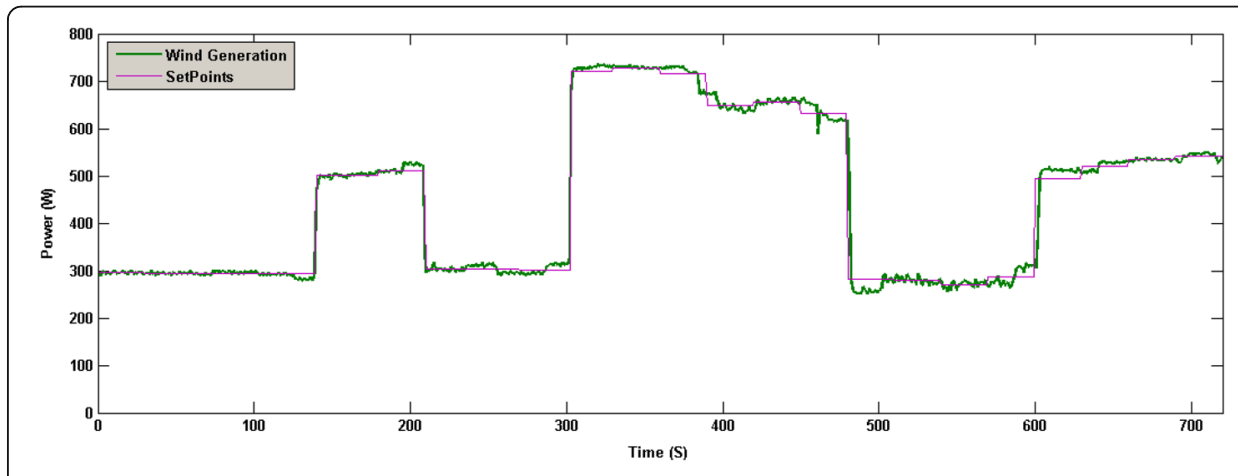

Fig. 9 Real-time results of wind turbine emulator in BUS \#15 


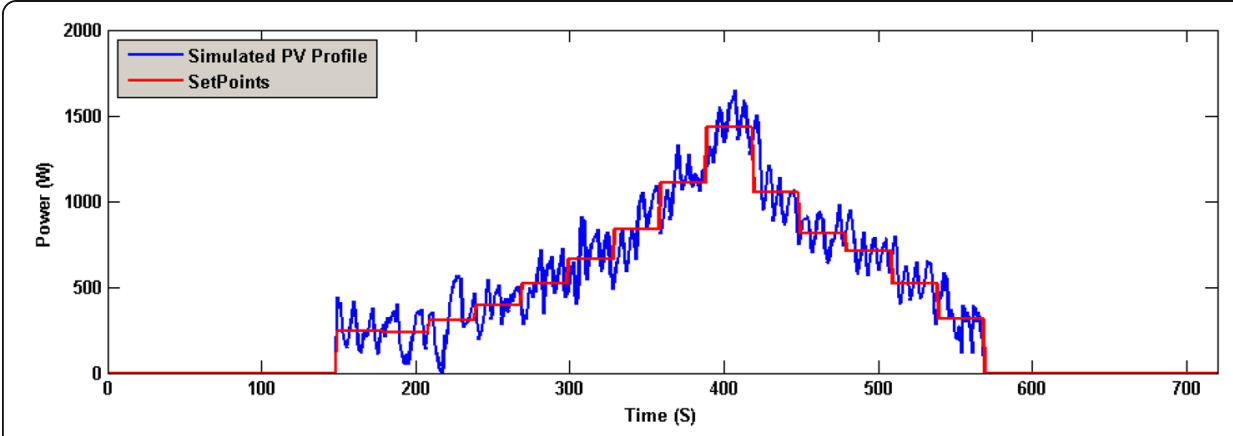

Fig. 10 Real-time results of PV emulator in BUS \#10

consumption changed, the loads require some time to reach the desired consumption level. This is one of the main differences between the experimental works and simulation works; in simulation environment the consumption rate changes immediately, however, consumers modeled by HIL devices employed in this platform require some time to meet the desired rate of consumption since it accommodates analog control in AC Loads.

Moreover, Fig. 9 illustrates the real-time wind generation of the prosumer in BUS \#15, which has been simulated by the $1.2 \mathrm{~kW}$ wind turbine emulator. The wind speed data provided by OP5600 to the wind turbine emulator has been acquired from (Meteo 2017).

As it can be seen in Fig. 9, the set points are the scheduled values that have been requested from the wind turbine to be emulated. Consequently, the emulator produces power and transmits the actual measurements of active power generation (green line in Fig. 9) to the OP5600. By this way, the system is able to emulate a scheduled wind generation profile based on the electrical grid conditions, such as voltage variations.

Figure 10 shows the real-time results of the PV emulator considered in BUS \#10. As it is clear in Fig. 10, the set points are the scheduled amount of power to be generated by PV emulator, and the simulated PV profile (blue line) is the real generated profile by the PV emulator, which has been transmitted to OP5600 in real-time. Also, as Fig. 10 illustrates, there are a lot of variations in generation curve, which is due to the voltage variations in the AC side of the inverter. In this condition, the controller section of the DC power supply attempted to keep the generation level on the desired generation level.

Additionally, Fig. 11 demonstrates the PV generation results of BUS \#5 in the CSP network. The results shown in this figure are the real production data of GECAD PV system with $10 \mathrm{~kW}$ capacity of generation. The considered generation profile is for an

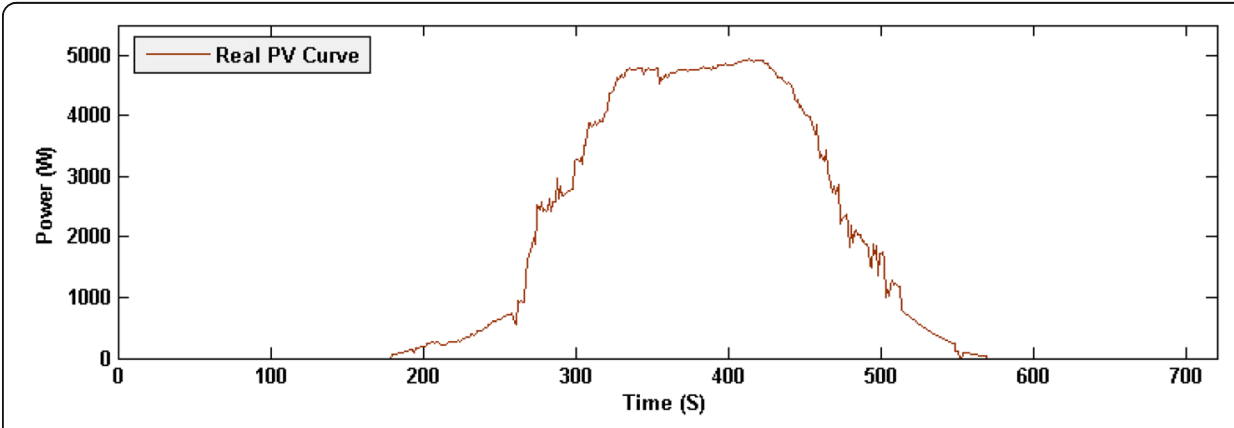

Fig. 11 Acquired results for real $10 \mathrm{~kW}$ PV producer considered in BUS \#5 


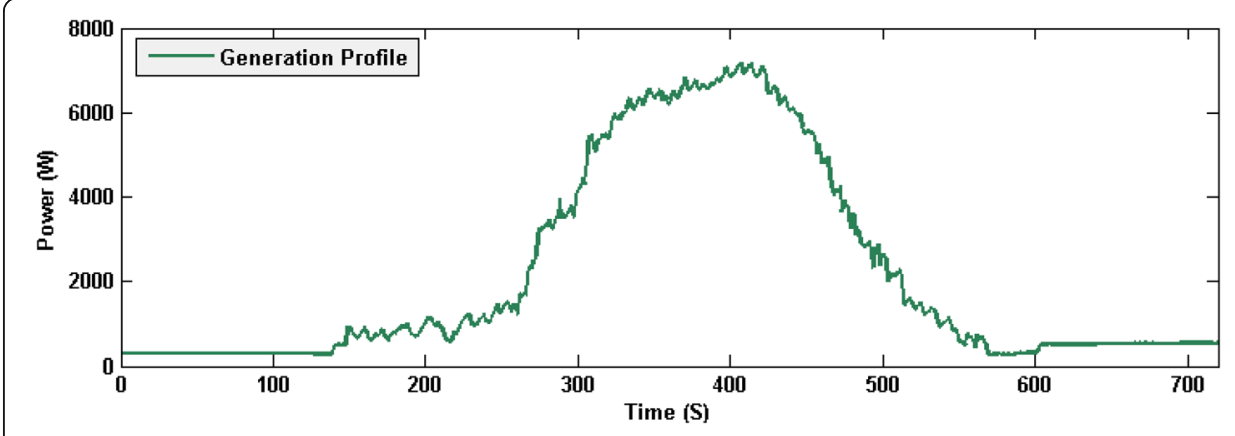

Fig. 12 Real-time profile of aggregated generation from BUS \#15,\#10, and \#5

entire day with $1 \mathrm{~min}$ time interval (1440 periods of $1 \mathrm{~min}$, for $24 \mathrm{~h}$ ) available in GECAD database, and the profile shown in Fig. 11 is the same profile; however, OP5600 acquires the data with $0.5 \mathrm{~s}$ time interval ( 1440 periods of $0.5 \mathrm{~s}$, in total $720 \mathrm{~s}$ ).

As a final result, Fig. 12 shows the total aggregated generation from BUS \#15, \#10, and \#5 in the CSP network in real-time, which is the sum of generation profiles shown in Figs. 9, 10 and 11.

The results shown in Fig. 12 are the actual measurements from the generation resources, which OP5600 acquired in real-time from different resources with different time intervals and merged into a unique profile. Since this is a real aggregated generation profile, it can be used by a CSP for several simulation purposes, such as remuneration processes or electricity market negotiations.

In sum, the results regarding the real-time simulation model of the CSP are illustrated in this section. These results prove the platform skills in order to validate a business model for optimal resource scheduling using several real and laboratory hardware resources and the real-time simulator.

\section{Conclusions}

The use of demand response programs and distributed energy resources, especially renewable generation, are a key role of nowadays distribution network. Moreover, in order to have an efficient solution for these resources management, third-party entities, namely curtailment service providers, are very relevant in this scope. The model presented in this paper concerns the real-time simulation of a curtailment service provider by utilizing several real and laboratory hardware resources. All the equipment presented in this paper has been employed in the real-time simulator as hardware-in-the-loop in order to take advantage of realistic results inside of the simulation environment. Moreover, an optimization problem is developed in this paper, which enables the curtailment service provider to have an optimal solution for the resource scheduling with the aim of minimizing the operation costs.

The presented case study tested and validated the system capabilities in terms of controlling and monitoring the real resources from the simulation environment by using a real-time simulator. The results of the case study are the ones actually measured from the real and laboratory loads and generators. These results demonstrate that the real implementation of management scenarios, namely demand response programs or resources scheduling, electrical grid conditions play a key role since voltage variations 
affect the consumption and generation profiles. Moreover, it is shown that the results obtained from the optimization have a little difference compared to the real-time simulation results. This is because of the reaction of the real resources outside of simulation environment; real resources take some time in order to fulfill the system goal and reach the desired consumption or generation level. In fact, this issue validates the need for a real-time simulation and hardware-in-the-loop methodology using real hardware resources, before massive implementation of business models.

\section{Nomenclature}

Parameters

OCOperational Costs of CSP

$P_{E x t}$ Incoming power from external suppliers

$P_{D G}$ Produced power of DG

$P_{D L C}$ Power reduction in scope of DLC DR program

$P_{\text {Red }}$ Power reduction in scope of Red. DR program

$P_{R T P}$ Power reduction in scope of RTP DR program

$C_{E x t}$ Cost of purchased power from external suppliers

$C_{D G}$ Cost of produced DG power

$C_{D L C}$ Cost of remuneration for DLC DR program

$C_{R e d}$ Cost of remuneration for Red. DR program

$C_{R T P}$ Cost of remuneration for RTP DR program

$P_{\text {Load }}$ The total power consumption of CSP

$P_{\text {Cons }}$ Consumption of each CSP player

$P_{E x t}^{\max }$ Maximum capacity of incoming power from external suppliers

$P_{D G}^{\max }$ Maximum generation capacity of DG

$P_{D L C}^{\max }$ Maximum reduction for DLC program

$P_{R e d}^{\max }$ Maximum reduction for Red. Program

$P_{R T P}^{m a x}$ Maximum reduction for RTP program

Indexes

INumber of time periods

$S$ Number of external suppliers

CsNumber of CSP customers

Abbreviations

CSP: Curtailment service provider; DG: Distributed Generation; DLC: Direct Load Control; DR: Demand response; DRER: Distributed renewable energy resources; HIL: Hardware-in-the-loop; PV: Photovoltaic; RTP: Real-Time Pricing; VPP: Virtual power player

\section{Funding}

The present work was done and funded in the scope of the following project: NetEfficity Project (P2020 - 18015), co-funded by Portugal 2020, Fundo Europeu de Desenvolvimento Regional (FEDER) through Programa Operacional Competitividade e Internacionalização (PO CI); and UID/EEA/00760/2013 funded by FEDER Funds through COMPETE pro-gram and by National Funds through FCT.

Availability of data and materials

Please contact author for data requests.

Authors' contributions

OA organized the real-time simulation models and optimization problems and drafted the manuscript. PF carried out the overall concepts of CSP and demand response programs designing. ZV raised and developed the overall idea of the work. All authors read and approved the final manuscript. 
Ethics approval and consent to participate

Not applicable.

\section{Consent for publication}

Not applicable.

\section{Competing interests}

The authors declare that they have no competing interests.

\section{Publisher's Note}

Springer Nature remains neutral with regard to jurisdictional claims in published maps and institutional affiliations.

Received: 10 January 2018 Accepted: 11 June 2018

Published online: 26 July 2018

\section{References}

Abrishambaf O, Faria P, Gomes L, Spínola J, Vale Z, Corchado J (2017) Implementation of a real-time microgrid simulation platform based on centralized and distributed management. Energies 10(6):806-820

Abrishambaf O, Gomes L, Faria P, Vale Z (2015) Simulation and control of consumption and generation of hardware resources in microgrid real-time digital simulator. Paper presented at IEEE PES innovative smart grid technologies Latin America (ISGT LATAM), Montevideo, 5-7

Aghaei J, Alizadeh M (2013) Demand response in smart electricity grids equipped with renewable energy sources: a review. Renew Sust Energ Rev 18:64-72

Alvarez-Gonzalez F, Griffo A, Sen B, Wang J (2017) Real-time hardware-in-the-loop simulation of permanent-magnet synchronous motor drives under Stator faults. IEEE Trans Ind Electron 64(9):6960-6969

Bakr S, Cranefield S (2015) Using the Shapley value for fair consumer compensation in energy demand response programs: comparing algorithms. Paper presented at IEEE International Conference on Data Science and Data Intensive Systems, Sydney, 11-13

Bottaccioli L, Estebsari A, Patti E, Pons E, Acquaviva A (2017a) A novel integrated real-time simulation platform for assessing photovoltaic penetration impacts in smart grids. Energy Procedia 111:780-789

Bottaccioli L, Estebsari A, Pons E, Bompard E, Macii E, Patti E, Acquaviva A (2017b) A flexible distributed infrastructure for real-time Cosimulations in smart grids. IEEE Trans Industr Inform 13(6):3265-3274

Falvo M, Graditi G, Siano P (2014) Electric vehicles integration in demand response programs. Paper presented at 2014 International Symposium On Power Electronics, Electrical Drives, Automation And Motion, Ischia, Italy, 18-20

Faria P, Vale Z (2013) Demand response programs definition using demand price elasticity to define consumers aggregation for an improved remuneration structure. Paper presented at IEEE PES ISGT Europe, Lyngby, 6-9

Faria P, Vale Z, Baptista J (2015) Constrained consumption shifting management in the distributed energy resources scheduling considering demand response. Energy Convers Manag 93:309-320

Gkatzikis L, Koutsopoulos I, Salonidis T (2013) The role of aggregators in smart grid demand response markets. IEEE J Sel Areas Commun 31(7):1247-1257

Hillman T (2011) Can demand response receive comparable treatment to traditional generation? 2011 IEEE/PES Power Systems Conference and Exposition, Phoenix, pp 20-23

Hurtado L, Mocanu E, Nguyen P, Gibescu M, Kamphuis R (2018) Enabling cooperative behavior for building demand response based on extended joint action learning. IEEE Trans Industr Inform 14(1):127-136

Khezeli K, Lin W, Bitar E (2017) Learning to buy (and sell) demand response. IFAC-Papers Online 50(1):6761-6767

Li C, Chen Y, Luo F, Xu Z, Zheng Y (2017) Real-time decision making model for thermostatically controlled load aggregators by natural aggregation algorithm. Paper presented at IEEE International Conference On Energy Internet (ICEI), Beijing, 17-21

Mao C, Leng F, Li J, Zhang S, Zhang L, Mo R, Wang D, Zeng J, Chen X, An R, Zhao Y (2018) A 400-V/50-kVA digitalphysical hybrid real-time simulation platform for power systems. IEEE Trans Ind Electron 65(5):3666-3676

Marulanda G, Valenzuela J, Salazar H (2014) An assessment of the impact of a demand response program on the Colombian day-ahead electricity market. Paper presented at IEEE PES Transmission \& Distribution Conference and Exposition - Latin America (PES T\&D-LA), Medellin, 10-13

ISEP Meteo (2017) http://meteo.isep.ipp.pt/weather/index.html. Accessed 19 Dec 2017

Mortaji H, Hock Ow S, Moghavvemi M, Almurib H (2017) Load shedding and smart-direct load control using internet of things in smart grid demand response management. IEEE Trans Ind Appl 53(6):5155-5163

Olivares D, Mehrizi-Sani A, Etemadi A, Canizares C, Iravani R, Kazerani M et al (2014) Trends in microgrid control. IEEE Trans Smart Grid 5(4):1905-1919

Paterakis N, Erdinç O, Catalão J (2017) An overview of demand response: key-elements and international experience. Renew Sust Energ Rev 69:871-891

Reddy S (2016) Optimizing energy and demand response programs using multi-objective optimization. Electr Eng 99(1):397-406

Rotger-Griful S, Chatzivasileiadis S, Jacobsen R, Stewart E, Domingo J, Wetter M (2016) Hardware-in-the-loop co-simulation of distribution grid for demand response. Paper presented at Power Systems Computation Conference (PSCC), Genoa, 20-24

Shariatzadeh F, Mandal P, Srivastava A (2015) Demand response for sustainable energy systems: a review, application and implementation strategy. Renew Sust Energ Rev 45:343-350

Siano P (2017) Demand response and smart grids-a survey. Renew Sust Energ Rev 30:461-478

Silva M, Fernandes F, Morais H, Ramos S, Vale Z (2015) Hour-ahead energy resource management in university campus microgrid. Paper presented at IEEE Eindhoven Powertech, Eindhoven, 29 June-2 July 20

Wang Q, Zhang C, Ding Y, Xydis G, Wang J, Østergaard J (2015) Review of real-time electricity markets for integrating distributed energy resources and demand response. Appl Energy 138:695-706 